\title{
Fuzzy Logic Based Path Tracking Controller for Wheeled Mobile Robots
}

\author{
Umar Farooq, K. M. Hasan, Athar Hanif, Muhammad Amar, and Muhammad Usman Asad
}

\begin{abstract}
This paper describes the design and implementation of a path tracking controller based on fuzzy logic to aid in mobile robot navigation in indoor environments. The proposed controller consists of two stages. The controller at first stage called 'Orientation Error Controller' positions the robot perpendicular to the desired path and the controller at second stage called 'Distance Error Controller' minimizes the perpendicular distance between the robot and desired path. The two controllers are triggered in sequence to follow the desired trajectory. The robot with the designed controller is tested in indoor environments with various desired paths as its input and is found to follow the required paths successfully which proves the validity of the controller.
\end{abstract}

Index Terms - Path tracking, fuzzy logic, orientation error controller, distance error controller, AT89C52 microcontroller implementation of fuzzy logic.

\section{INTRODUCTION}

In order to navigate in an environment, the robot must generate a collision free path from source to destination. However, if the path is known in advance, the robot can track the path to reach the destination. The path tracking can be achieved with linear or non linear feedback control methods such as described in [1]. These methods require an accurate kinematics or dynamics model, which in most cases is not possible due to the noisy and unpredictable environment. Therefore, some intelligent techniques are required which can handle these uncertainties and cope up with complex situations to enable the mobile robot reach the destination with minimum possible error. Amongst these techniques, fuzzy logic presents a promising solution since it does not require the complex mathematical model and uses human experience to make inferences even under uncertainty [2].

This paper discusses an experimental fuzzy logic controller for mobile robot path tracking in indoor environments. The controller is comprised of two sub-controllers namely 'Orientation Error Controller (OEC)' and 'Distance Error Controller (DEC)'. The task of OEC is to position the robot perpendicular to the path while the task of DEC is to minimize the distance error between the desired path and robot current position and hence make the robot to follow the desired path.

Manuscript received May 27, 2013; revised August 22, 2013.

Umar Farooq, Muhammad Amar, and Muhammad Usman Asad are with Department of Electrical Engineering, University of The Punjab Lahore (e-mail: engr.umarfarooq@yahoo.com, amar.ete611@yahoo.com, usmanasad01@hotmail.com).

K. M. Hasan is with Department of Electrical Engineering, University of Engineering \& Technology Lahore (e-mail: kmhasan@uet.edu.pk).

Athar Hanif is with Department of Electrical Engineering, The University of Lahore (e-mail: athar.hanif@ee.uol.edu.pk).
The controllers are designed using fuzzy logic tool box of MATLAB ${ }^{\circledR}$ and implemented in real time with low cost and readily available AT89C52 microcontroller. Experimental results have shown the validity of designed controller in tracking a desired path.

\section{RELATED WORK}

Many techniques utilizing fuzzy logic have been proposed in literature for path tracking behavior of mobile robots. In [3], design of fuzzy logic controller for path tracking of differentially driven mobile robot is presented. The inputs to the controller are position and orientation errors (described with five linguistic terms) with respect to the reference path while the output of the controller is the desired heading direction with respect to the current heading (also expressed with five linguistic terms). Simulation of proposed controller is carried out on two paths namely cubic splines and circle and results have shown the validity of the designed controller in tracking these paths with a position error within $50 \mathrm{~mm}$ and orientation error within 7 degrees in case of cubic splines and a position error within 10mm and orientation error within 6 degrees in case of circle type path. In [4], mobile robot path tracking problem is solved by using 5 layer fuzzy neural network (FNN) trained with back error propagation algorithm with additive momentum. The inputs to FNN are extracted from theorem of line between two points described as error in perpendicular distance of robot from desired trajectory and error in orientation with respect to the desired trajectory while the outputs are linear and angular velocities of the robot. The robot is also provided with obstacle avoidance control behavior based on fuzzy logic for collision avoidance during path tracking. The controllers are finally tested by carrying out computer simulations in environments containing both stationary and moving obstacles and tracking error is found to lie below $2 \mathrm{~mm}$ which shows the effectiveness of the proposed controllers. In [5], two fuzzy logic controllers named as robot positioning controller (RPC) and robot following controller (RFC) are designed for navigation of car-like mobile robot between two points. RPC ensures the accurate positioning of the robot at destination while RFC ensures the accurate orientation of robot at desired destination. Error in position of robot with respect to intermediate point called 'sub-goal' and error in orientation with respect to sub-goal form inputs to RPC which moves the robot to sub-goal position by minimizing these errors. Once the robot is positioned on sub-goal, second FLC (RFC) which takes on its input the error in position with respect to goal location and error in orientation with respect to final location drives the robot on a circular trajectory by following a virtual mobile robot to goal 
position with desired orientation. To show the validity of proposed controllers, simulations are carried out in Matlab 6.5 followed by the real time implementation on Robucar, a nonholonomic mobile robot designed by INRIA (France) and manufactured by Robosoft. Results have shown the effectiveness of controllers for indoor as well as outdoor environments. In [6], mobile robot navigation task is performed with the help of three fuzzy logic controllers implementing the behaviors 'reach the target', 'avoid obstacles' and 'explore the environment'. 'Reach the target' behavior captures information from camera mounted on the ceiling and calculates the distance to target and alignment error with target. It is the primary behavior for robot navigation which first aligns the robot with target and then moves the robot in straight line towards the target. To avoid hurdles in its way, mobile robot is provided with 'obstacle avoidance' behavior which takes inputs from eight IR sensors mounted on periphery of robot and ensures collision free motion amongst obstacles. However this behavior does not ensure that the robot will not get stuck in box canyons. To overcome this problem, 'explore the environment' behavior is introduced which makes the robot mark regions already visited and looks for unexplored areas. A fuzzy supervisor combines information from three behaviors and generates speed commands for mobile robot following a priority code. To prove the validity of controllers, experiments are carried out on differentially driven mobile robot named 'Khepra' in a $1.2 \times 1.2 \mathrm{~m}^{2}$ arena, viewed entirely by the webcam. All the processing is done on a dSPACE board interfaced through MATLAB environment. After a number of experiments, a tradeoff is found between the robot speed (avoid the obstacle priority) and smoothness of the paths traveled (reach the target priority) in fuzzy supervisor and satisfactory results are obtained with this tuned fuzzy supervisor. In [7], a fuzzy logic behavior based navigation scheme called 'minimum risk method' and grid based map model called 'memory grid' is presented for mobile robot navigation in unknown, cluttered indoor environments with dead ends. Behavior based navigation combines information from three behaviors namely 'obstacle avoidance (OA), 'path searching (PS)' and 'goal seeking (GS)' according to a weight criterion along with features extracted from 'memory grid' which keeps track of obstacle locations and robot trajectory and drives the robot towards the safest region with minimum risk. The scheme is compared with already existing models for robot navigation and is verified from both simulation and real world experiments. The only draw back in this scheme relates to the odometery errors as the robot is not designed for long distance and outdoor navigation.

\section{PROBLEM FORMULATION}

The path tracking problem is depicted in Fig. 1 where $\Delta \theta$ indicates the orientation error of the robot with respect to the desired path and $\Delta d$ denotes the perpendicular distance error between robot and desired path. The task of the controller now is to reduce both these errors to zero to ensure the robot positioned on the desired trajectory. Thus, two fuzzy logic controllers are designed in order to reach and track the desired path. The first controller known as 'Orientation Error Controller' aligns the robot with the target and takes the error between the goal angle and current robot angle as input. The outputs from the controller are right motor and left motor speeds. The second fuzzy controller known as 'Distance Error Controller' minimizes the perpendicular distance of robot from the desired path to reach the desired path and track it. It takes orientation error and perpendicular distance error as inputs and generates control commands for right and left motors.

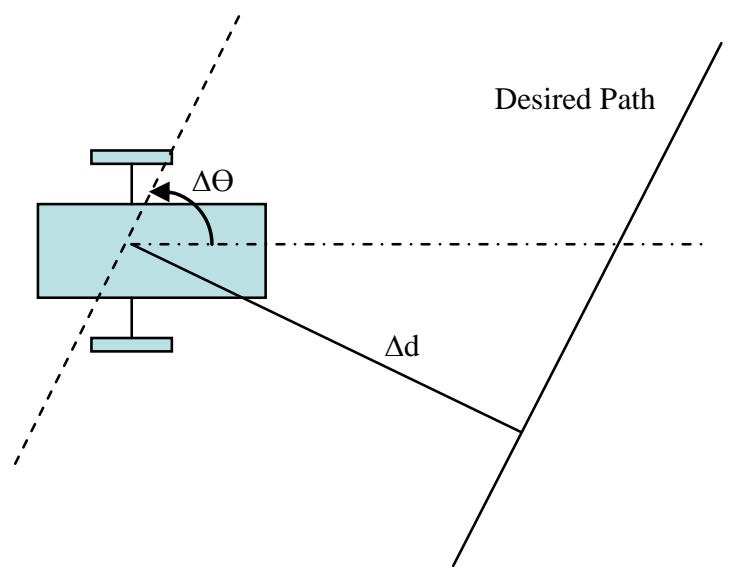

Fig. 1. Path tracking problem description

\section{FUZZY CONTROLLER DESIGN}

MATLAB ${ }^{\circledR}$ Fuzzy Logic Toolbox is used to aid in fuzzy logic controllers (FLC) design [8]. The steps in FLC design are described in this section.

\section{A. Fuzzification}

The input to FLC1 i.e., 'Orientation Error Controller' is described by three fuzzy sets, Small, Med and Large; the universe of discourse being from zero (minimum) to hundred (maximum). The output of FLC1 i.e., left motor speed (LM) and right motor speed (RM) is also described by three linguistic values: Slow, Med and Fast; the universe of discourse being from zero (minimum) to hundred (maximum) which describes the duty cycle of PWM signal. Similarly, the inputs to FLC2 i.e., 'Distance Error Controller' are described by three linguistic variables namely Small, Med and Large and outputs are also described by three linguistic variables namely Slow, Med and Fast. All the linguistic variables are described by triangular membership function, shown in Fig. 2. Triangular membership function (MF) is selected because of limited computational resources of microcontroller.

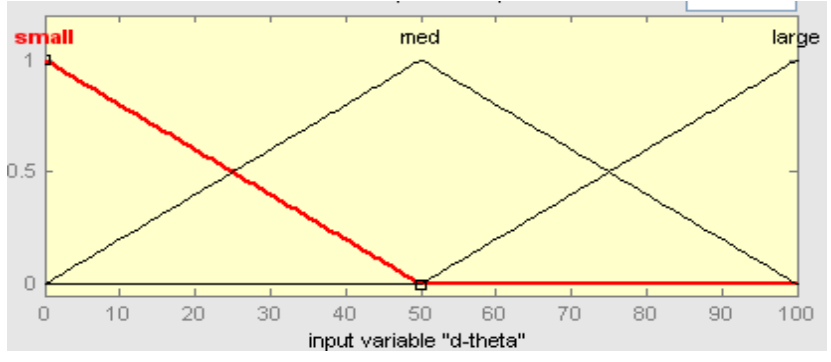

(a) 


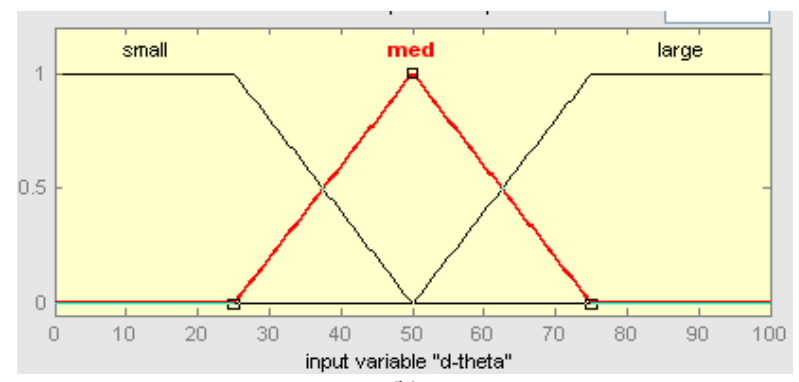

(b)

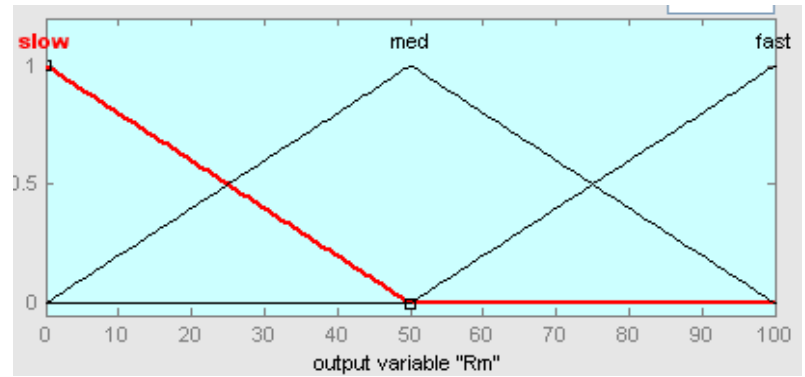

(c)

Fig. 2. Path tracking controller membership function (MF) description (a) input MF for FLC1 (b) input MF for FLC2 (c) output MF for FLC1 and FLC2

\section{B. Fuzzy Rule Base}

'Orientation Error Controller' operates on three rules which establish a relation between the orientation error of robot with respect to desired path and speed of left and right motors. These rules are listed below:

- IF delta-theta $(\Delta \theta)$ is LARGE THEN Right motor (Rm) is SLOW and Left motor (Lm) is MEDIUM

- IF delta-theta $(\triangle \theta)$ is MEDIUM THEN Right motor (Rm) is MEDIUM and Left motor (Lm) is MEDIUM

- IF delta-theta $(\triangle \Theta)$ is SMALL THEN Right motor (Rm) is FAST and Left motor (Lm) is FAST

The variations in right and left motors speeds given the orientation error are shown in Fig. 3. It can be seen from the figure that motor speeds vary smoothly over the entire orientation error range.

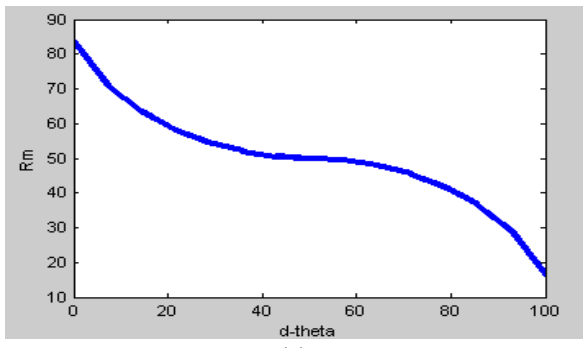

(a)

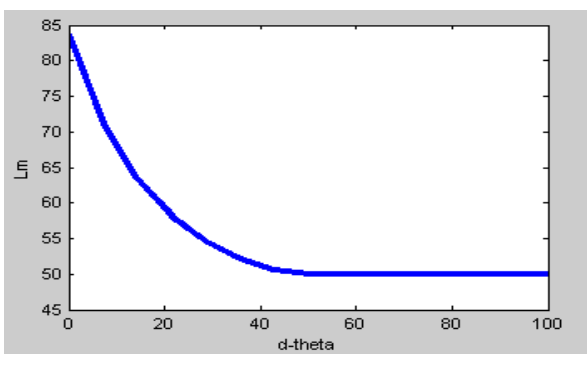

(b)

Fig. 3. Motor response for orientation error controller (a) right motor speed variation (b) left motor speed variation

For 'Distance Error Controller', a total of nine rules are designed. These rules map the orientation and distance errors to motor speeds in terms of linguistic values. A control surface is then plotted to visualize the variation in motor speeds given the error values. The rule base for right and left motor speed control is shown in Table I and Table II respectively while control surface is shown in Fig. 4.

TABLE I: Rule BASE For ChANGE IN Right MOTOR SPEED FOR DistANCE ERROR CONTROLLER

\begin{tabular}{cccc}
\hline \hline$\Delta \Theta / \Delta \mathrm{d}$ & Large & Med & Small \\
Large & Fast & Fast & Fast \\
Med & Med & Med & Fast \\
Small & Slow & Slow & Slow \\
\hline \hline
\end{tabular}

TABLE II: RulE BASE FOR CHANGE IN LEFT MOTOR SPEED FOR DiSTANCE

\begin{tabular}{clll} 
& \multicolumn{3}{l}{ ERROR CONTROLLER } \\
\hline \hline L $/ \Delta \mathrm{d}$ & Large & Med & Small \\
Large & Fast & Med & Slow \\
Med & Fast & Fast & Fast \\
Small & Fast & Fast & Fast \\
\hline \hline
\end{tabular}

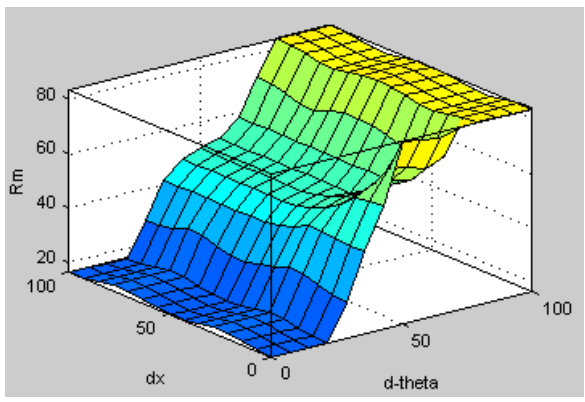

(a)

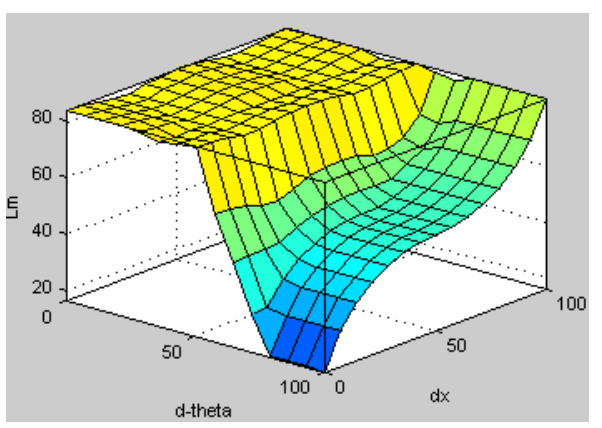

(b)

Fig. 4. Motor Response for Distance Error Controller (a) Right Motor Control Surface (b) Left Motor Control Surface

\section{Fuzzy Implication}

Fuzzy implication helps to evaluate the consequent part of each rule. Among the various implication methods available in literature, Mamdani implication method is selected. After the inputs have been fuzzified and FLC know the degree to which each part of the antecedent of a rule has been satisfied, degree of fulfillment of (DOF) of each rule is calculated using AND operator. The output membership function is then truncated at DOF level. All the rules are evaluated in this 
manner and final output membership functions are aggregated in a cumulative manner using OR operator to yield the final fuzzy output. An example of the implication process for FLC1 is shown in Fig. 5 (a) where small orientation error results in fast motion of the robot while implication process for FLC2 is shown in Fig. 5 (b) which corresponds to a situation in which the robot is positioned in parallel to the desired path at large perpendicular distance (The path is towards right side of the robot). In this case, robot should move fast towards right to minimize the perpendicular distance.

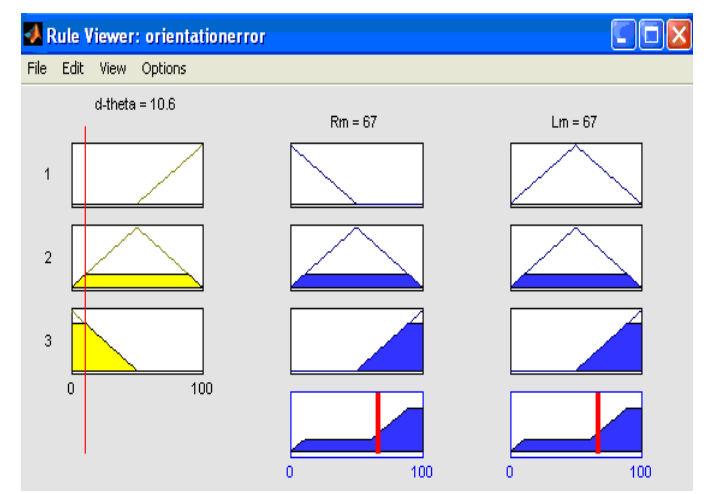

(a)

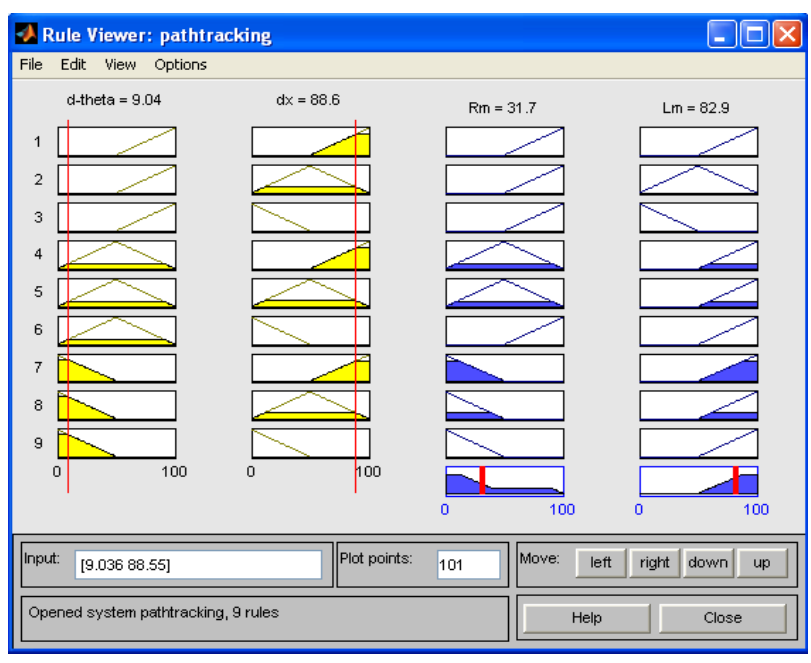

(b)

Fig. 5. Fuzzy Implication and Defuzzification Process for (a) Orientation Error Controller (b) Distance Error Controller

\section{Defuzzification}

The result of the implication and aggregation step is the fuzzy output, which is the union of all the individual rules that are validated or fired. Conversion of this fuzzy output to crisp output is defined as defuzzification. Commonly used defuzzification methods are maximum defuzzification and centroid defuzzification. COA method has been used for this purpose and is described as:

$$
Z_{o}=\frac{\sum_{i=1}^{n} Z_{i} \mu_{\text {out }}\left(Z_{i}\right)}{\sum_{i=1}^{n} \mu_{\text {out }}\left(Z_{i}\right)}
$$

where $\mu_{\text {out }}\left(Z_{i}\right)$ are the $i=1,2, \ldots, n$ sampled values of the aggregated output membership function and $Z_{o}$ is the crisp value which describes the duty cycle of PWM signal for controlling the speed of motors.

\section{EXPERIMENTAL PLATFORM}

The experimental prototype, shown in Fig. 6, is a rectangular shaped differentially steered mobile robot. Two dc motors independently control two wheels on a common axis. Two caster wheels are provided for support. Five SRF05 ultrasonic sensors, two wheel encoders based on QRD1114 reflective sensors and a digital compass fabricated using needle-like compass comprise the sensory system of the robot. A microcontroller board incorporating AT89C52 microcontrollers is designed to run fuzzy controllers. To drive the dc motors from microcontroller, a motor interface board is designed using 4N25 and L298N integrated circuit chips.

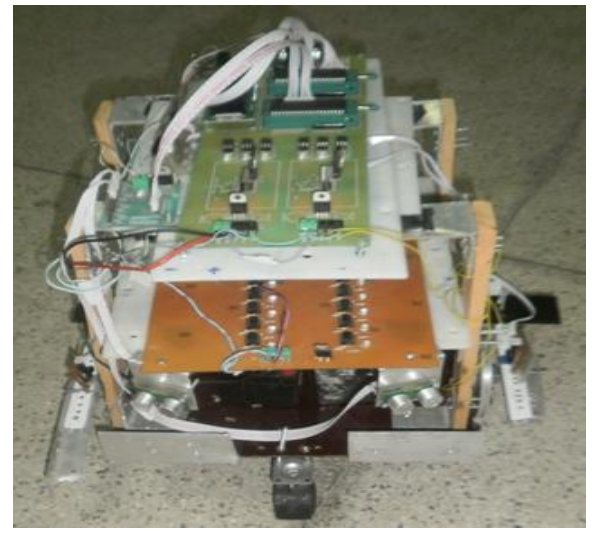

Fig. 6. Experimental Prototype

\section{FUZZY CONTROLLER IMPLEMENTATION}

Both fuzzy logic controllers are implemented using a single chip AT89C52 microcontroller having 256 bytes of RAM and $8 \mathrm{~Kb}$ of program memory and are triggered in sequence. Orientation Error Controller positions the robot perpendicular to the desired path while Distance Error Controller minimizes the perpendicular distance between the robot and desired path to enable the robot follow the desired path. The flow chart for program execution is shown in Fig. 7.

\section{EXPERIMENTAL RESULTS}

Path tracking controller is tested with various initial and final positions of robot in indoor environments. The controller first orients the robot perpendicular to the desired path and then minimizes the distance to track the path. Fig. 8 shows the steps taken by the robot to follow the straight line trajectory at a distance of $60 \mathrm{~cm}$. The robot is positioned at an angle of 135 degress with reference to positive $\mathrm{x}$-axis. Thus OEC orients the robot in counter clockwise direction covering an additional angle of 135 degrees to place it perpendicular to the path (Fig. 8 (a)-(f)). DEC then minimizes the error in $\mathrm{x}$-component of distance $(\mathrm{dx})$ as well as error in angle $(\mathrm{d} \theta)$ in a smooth fashion to reach the desired path (Fig. 8 (g)-(l)). When both the errors are small, the robot will follow the trajectory at fast speed. 


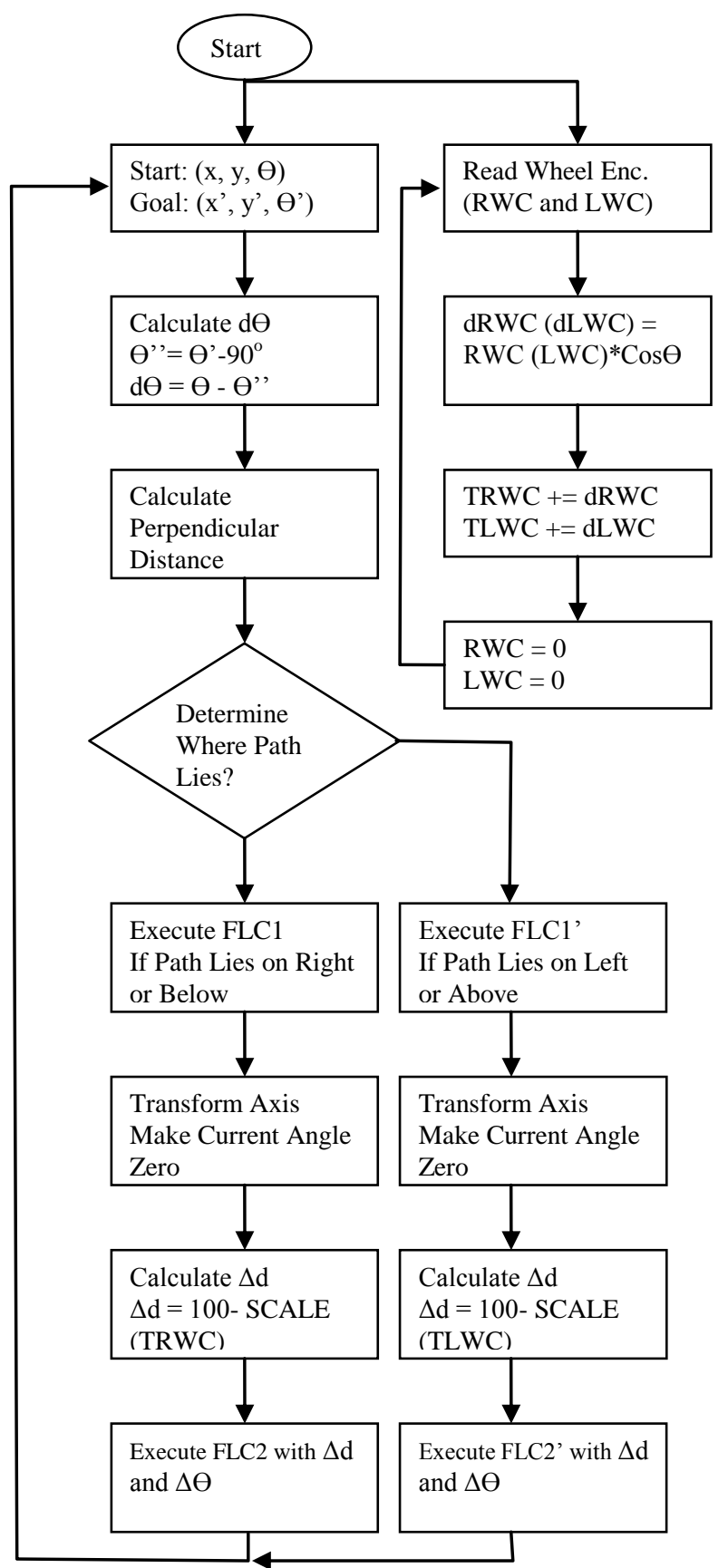

Fig. 7. Flow chart for program execution

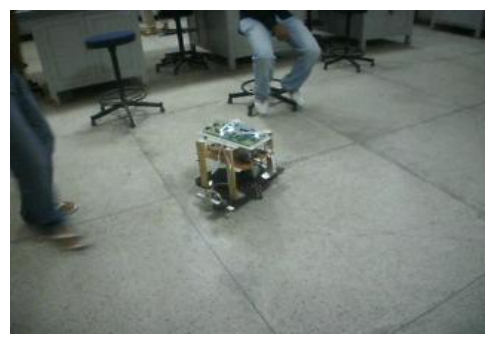

(a)

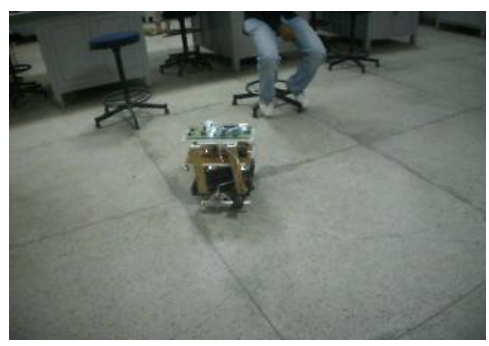

(b)

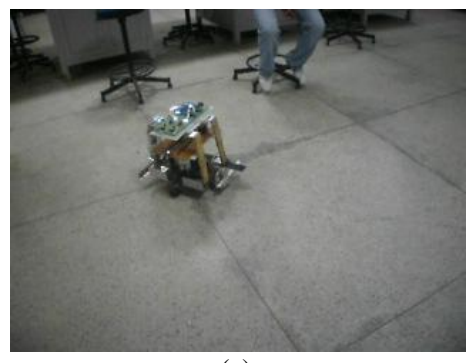

(c)

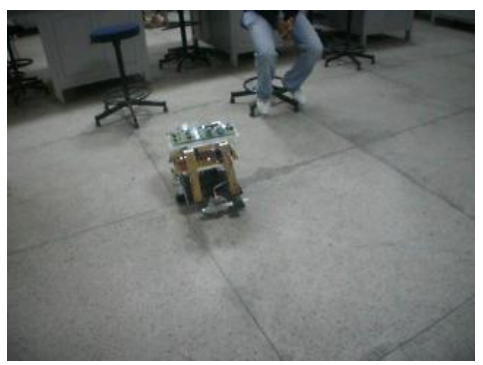

(d)

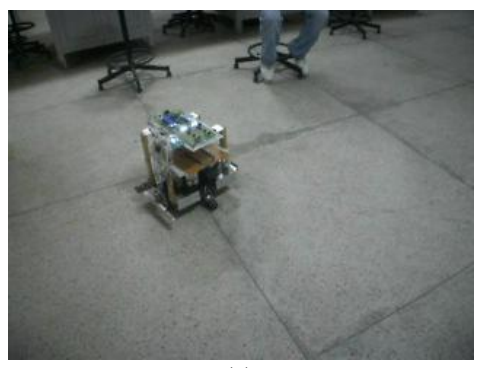

(e)

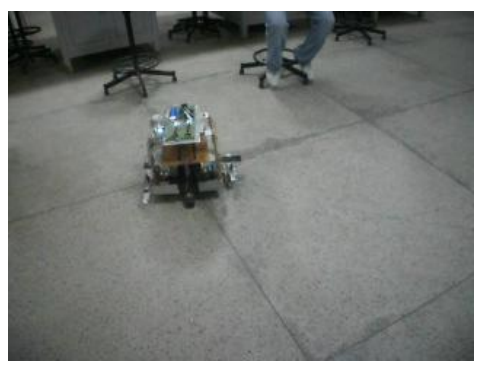

(f)

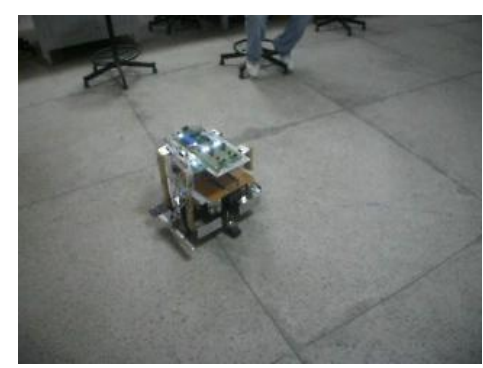

(g)

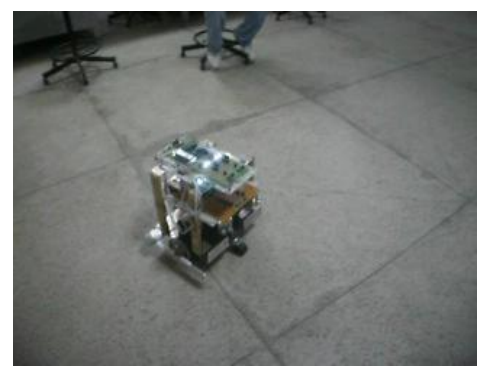

(h) 


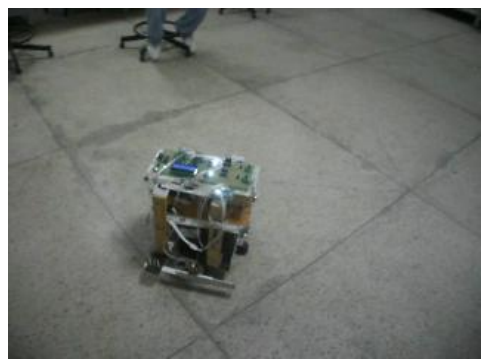

(i)

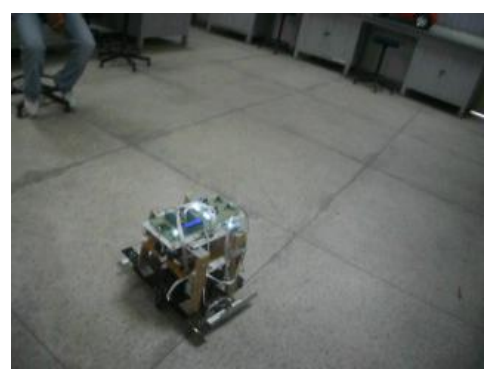

(j)

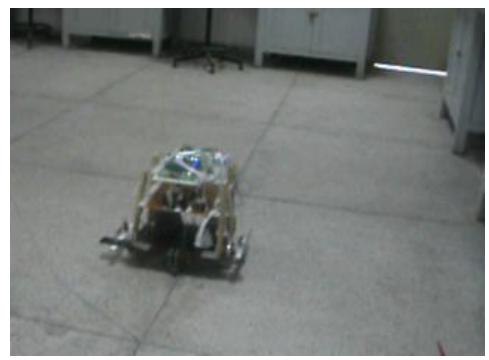

(k)

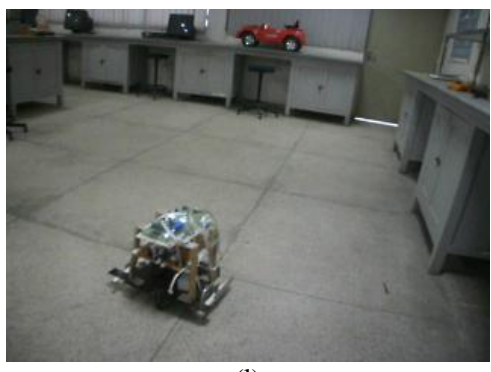

(1)

Fig. 8. Path tracking controller testing (a)-(f) Orientation Error Controller (g)-(1) Distance Error Controller

\section{CONCLUSIONS}

This work presents a fuzzy logic based controller for path tracking problem in differentially steered mobile robots. The controller can track the planned paths by considering them as sequence of connected straight lines. The present controller has performed well in indoor environments. However, it can be enhanced by introducing hurdle avoidance behavior which will enable the mobile robot to avoid obstacles, if exists on planned paths.

\section{REFERENCES}

[1] F. Mnif and F. Touati, "An adaptive control scheme for nonholonomic mobile robot with parametric uncertainty," International Journal of Advance Robotic Systems, vol. 2, no. 1, pp. 59-63, 2005.

[2] A. Saffiotti, "The uses of fuzzy logic in autonomous robot navigation: A catalog raisonne," Soft Computing, vol. 1, no. 4, pp. 180-197, 1997

[3] B. Lakehal, Y. Amirat, and J. Pontnau, "Fuzzy steering control of mobile robot," in Proc. IEEE/IAS International Conference on
Industrial Automation and Control: Emerging Technologies, 1995, pp. 383-386.

[4] Z. J. Du and Z. M. Wang, "Mobile robot path tracking in unknown dynamic environment," in Proc. IEEE RAM, 2008, pp.1201-1205.

[5] N. Ouadah, L. Ouark, and F. Boudjema, "Car-Like mobile robot oriented positioning by fuzzy controllers," International Journal of Advanced Robotic Systems, vol. 5, no. 3, pp. 249-256, 2008

[6] F. Cupertino, V. Giordano, D. Naso, and L. Delfine, "Fuzzy control of a mobile robot: implementing using a Matlab-based rapid prototyping system," IEEE Robotics \& Automation Magazine, vol. 13, no. 4, pp. 74-81, Dec 2006.

[7] M. Wang and J. N. K. Liu, "Fuzzy logic based real time robot navigation in unknown environments with dead ends," Robotics and Autonomous Systems, vol. 56, pp. 625-643. 2008

[8] J. S. Roger Jang and N. Gulley, MATLAB Fuzzy Logic Toolbox: A User's Guide, Mathworks Inc., 1997.

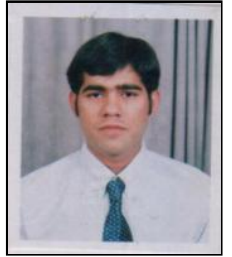

Umar Farooq did his B.Sc. and M.Sc. both in Electrical Engineering from University of Engineering \& Technology Lahore in 2004 and 2010 respectively. $\mathrm{He}$ is currently with the Department of Electrical Engineering, University of The Punjab Lahore. His research interests include the application of intelligent techniques to problems in control engineering, robotics and power electronics.

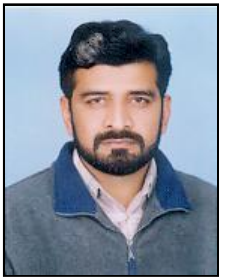

machine drives
K. M. Hasan did his B.Sc. in Electrical Engineering from University of Engineering \& Technology Lahore in 1986 and Ph.D. in Electrical Engineering from University of Bradford U.K. in 1999. He is currently with Department of Electrical Engineering, University of Engineering \& Technology Lahore where he is Professor of Control Engineering. His research interests include the intelligent control of mobile and legged robots, power converters and electrical

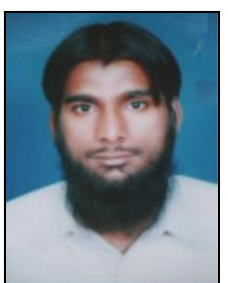

Athar Hanif holds B.Sc. and M.Sc. degrees in Electrical Engineering from University of Engineering \& Technology Taxila and University of Engineering \& Technology Lahore respectively. $\mathrm{He}$ is currently working towards the Ph.D. degree in Control Engineering from Muhammad Ali Jinnah University Islamabad. $\mathrm{He}$ is with Department of Electrical Engineering, The University of Lahore where he is working as Assistant Professor. His research interests include the robust nonlinear control of hybrid vehicles and power converters.

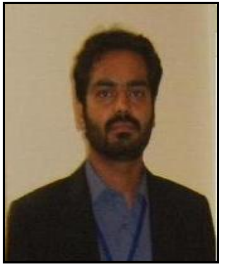

Muhammad Amar did his B.Sc. in Electrical Engineering from University of The Punjab Lahore in 2010 and M.Sc. in Electrical Engineering from University of Engineering \& Technology Lahore in 2012. He is currently working towards Ph.D. degree in Electrical Engineering from Monash University, Australia. His research interests include the application of intelligent techniques to problems in control engineering, robotics and machine vision.

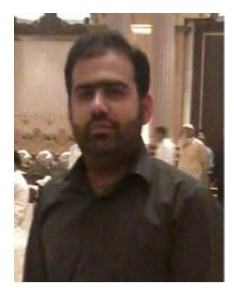

Muhammad Usman Asad did his B.Sc. in Electrica Engineering from University of The Punjab Lahore in 2010. He is working towards M.Sc. degree in Electrical Engineering from G.C. University Lahore $\mathrm{He}$ is with Department of Electrical Engineering, The University of Lahore where he is a Lecturer. His research interests include intelligent control of Robotics and Power systems. 